\title{
Evaluation Exhaustive De La Diversité Des Plantes Aromatiques Et Médicinales De La Péninsule Tingitane (Maroc)
}

\author{
Mohamed Ouziki \\ Institut des Techniciens Spécialisés en Agriculture, \\ Ben Karrich, Tétouan., Maroc

\section{Lahcen Taiqui} \\ Université Abdelmalek Essaadi, Faculté des sciences de Tétouan, \\ M’Hannech II, Tétouan, Maroc
}

doi: 10.19044/esj.2016.v12n15p210 URL:http://dx.doi.org/10.19044/esj.2016.v12n15p210

\section{Abstract}

Aromatic and Medicinal Plants (AMP) represent a large part of biodiversity. However, in the absence of a detailed record of this diversity, research and AMP development projects are generally limited to a small portion of ethnobotanical heritage. To guide prospection, exploitation and conservation studies of the existing potential, a comprehensive evaluation of the natural diversity of AMP is required. The western Rif region in northern Morocco, which area represents less than $1 \%$ of national territory, is home to about $50 \%$ of the Moroccan floristic richness (2053 species and infraspecific taxa). This heritage includes 630 taxa from which only 23 are currently exploited and merely $57 \%$ are recognized by ethno-botanists. Among these assets, a list is drawn up containing 106 taxa with a special conservation status (very rare or rare taxa, and / or endemic and / or threatened) and some of which are widely exploited. From a phytosociological point of view and based on an available database of 449 floristic samples, natural forest stands and matorrals contain about $27 \%$ of all MAP of the study area. These AMP organize almost half the floristic composition of sclerophyllous forests (oleaster, kermes oak and cork oak forest) and about a third of the richness of each of the other groups analyzed (thermophilic formations, pure fir, limestone mountains forest, calcifuges deciduous, maritime pine, matorrals). Mountain groups are the richest in exclusive, endemic, rare and endangered AMP.

Keywords: Diversity, flora, aromatic and medicinal plants, ethnobotany, plant sociology, conservation, Morocco 


\section{Résumé}

Les plantes aromatiques et médicinales (PAM) constituent une part importante de la diversité biologique. Cependant, en absence d'un inventaire détaillé de cette diversité, les recherches et les projets de valorisation des PAM sont généralement limités à un lot restreint d'espèces du patrimoine ethnobotanique. Pour contribuer à l'orientation des travaux de prospection et à la valorisation et conservation du potentiel existant, l'évaluation exhaustive de la diversité naturelle des PAM est nécessaire.

La région du Rif occidental au Nord du Maroc, d'une superficie inférieure à $1 \%$ par rapport au territoire national, abrite environ $50 \%$ de la richesse floristique marocaine (2053 espèces et taxons infra-spécifiques). Ce patrimoine englobe 630 taxons de PAM dont à peine 23 taxons sont actuellement très exploités et seulement $57 \%$ sont cités par les ethnobotanistes. Parmi ce patrimoine, une liste est établie contenant 106 taxons ayant un statut de conservation particulier (taxons très rares ou rares, ou/et endémiques ou/et menacés) et dont certains sont largement exploités.

Du point de vue phytosociologique, en se basant sur une base de données de 449 relevés floristiques disponibles, les groupements forestiers et matorrals naturels contiennent environ 27\% de l'ensemble des PAM de l'aire d'étude. Ces PAM constituent presque la moitié du cortège floristique des forêts feuillues sclérophylles (oléastraie, cocciféraie, subéraie) et environ le tiers de la richesse de chacun des autres groupements analysés (formations thermophiles, sapinière pure, forêts des montagnes calcaires, caducifoliés calcifuges, pinède maritime, matorrals). Les groupements de montagne sont les plus riches en PAM exclusives, endémiques, rares et menacées.

Mots Clés: Diversité, flore, Plantes aromatiques et médicinales, ethnobotanique, phytosociologie, conservation, Maroc.

\section{INTRODUCTION}

L’étude nationale sur la biodiversité au Maroc, effectuée par le Département de l'Environnement en 1997, estime la richesse de la flore vasculaire du pays à environ 4500 espèces et sous-espèces indigènes et naturalisées réparties entre 920 genres et 130 familles (Fennane, 2004), avec un taux d'endémisme qui atteint 978 taxons (El Oualidi et al, 2012). Cette richesse se répartit principalement dans deux grands hot spots méditerranéens: le Moyen et Haut-Atlas au centre du pays, et le complexe Bético-Rifain qui s'étend du nord du Maroc à l'Andalousie et au Tell Algérien (Medail \& Quézel, 1997). Dans ce dernier complexe, le Rif occidental, grâce à sa situation géographique, ses caractéristiques orographiques, lithologiques et bioclimatiques, se distingue par ses richesses naturelles particulièrement similaires à celles du Sud de l'Andalousie 
(Valdès, 1991). La végétation y est très diversifiée, les phytocénoses y sont luxuriantes et offrent les plus importantes potentialités forestières du Maroc (Benabid, 2002).

Les plantes aromatiques et médicinales (PAM), constituent une part importante de cette diversité biologique. Elles font partie, dans ces zones montagneuses, des ressources naturelles dont la population puise directement pour améliorer ses revenus et ne cessent de constituer l'essentiel de l'arsenal thérapeutique utilisé par les tradipraticiens pour soigner les gens (Bellakhdar, 2006). Cette dépendance vis-à-vis de ces ressources, en plus d'une demande de plus en plus accrue par des industries utilisatrices de ces plantes, s’ajoutent à l'intense dégradation des écosystèmes naturels et accentuent la menace de disparition d'un grand nombre de PAM. Cependant, malgré les différentes lois et stratégies élaborées par différents départements gouvernementaux, le développement de la filière des PAM dans une optique de conservation de la biodiversité reste très limité. En effet, à l'heure où les questions de biodiversité et de protection des espèces sont d'actualité, les gestionnaires de l'environnement, ne disposent pas toujours d'informations suffisantes pour établir des programmes d'exploitation, de protection et de conservation. Le manque de connaissance scientifique adéquate et de structures de gestion adaptée aux plantes aromatiques et médicinales, entrainent des risques inévitables de surexploitation qui peuvent compromettre leur conservation et leur utilisation durable et affecter divers services écologiques des écosystèmes qui les soutiennent. Cette situation porte également préjudice au progrès scientifique dans le domaine des ressources naturelles, dans la mesure où elle ne favorise pas des choix clairs quant aux axes de recherche prioritaires (Fennane \& Ibn Tattou, 1999).

On suppose que l'évaluation exhaustive de la diversité naturelle des PAM est nécessaire pour contribuer, d'une part, à l'orientation des travaux de prospection et de valorisation du potentiel existant et, d'autre part, à la gestion durable et à la conservation des espèces menacées. L’objectif est d'attirer l'attention des milieux de la recherche, de développement et des décideurs locaux sur la nécessité de reconnaître tout ce potentiel dans une optique d'encourager le développement de politiques publiques qui permettraient d'asseoir une viabilité écologique et socioéconomique de la filière des PAM.

Dans ce cadre, ce travail est consacré au développement d'une base de données de la richesse des PAM du Rif occidental, leur phytosociologie et leur statut de conservation (espèces rares, menacées et endémiques). En termes spécifiques, cette étude porte sur :

- la part occupée par la flore de PAM parmi la biodiversité floristique du territoire étudié;

- l'identification, parmi cette flore de PAM, des espèces rares, 
endémiques, menacées et celles exploitées à des fins ethnobotaniques.

- La caractérisation environnementale des PAM au sein des groupements végétaux naturels du territoire étudié.

\section{MATERIEL ET METHODES}

\section{1) Aire d'étude}

Elle correspond grossièrement à la Péninsule Tingitane située à l'extrémité Nord-Ouest du Maroc. En se référant à la subdivision floristique de Valdès et al. (2002), le territoire étudié couvre une superficie égale à environ $6686 \mathrm{~km}^{2}$ et correspond aux deux zones suivantes (figure 1) :

\section{- Le Rif Ouest :}

Cette aire de $2152 \mathrm{~km}^{2}$ occupe la partie Est de la péninsule de Tanger. Elle s'étend depuis la rivière Bouhya (affluent de l'oued Tighissas qui constitue la frontière avec l'aire de Targuist) jusqu'au Jbel Moussa sur le Détroit de Gibraltar. Elle est limitée au Sud par la région de Jebala.

\section{- Aire de Tanger :}

D’une superficie de $4534 \mathrm{~km}^{2}$, cette aire couvre la région depuis le Détroit de Gibraltar au Nord, jusqu'à la vallée du Loukkos au Sud. La côte Atlantique de Tanger à Asilah en constitue la limite Ouest, et la dorsale du Rif la limite Est.

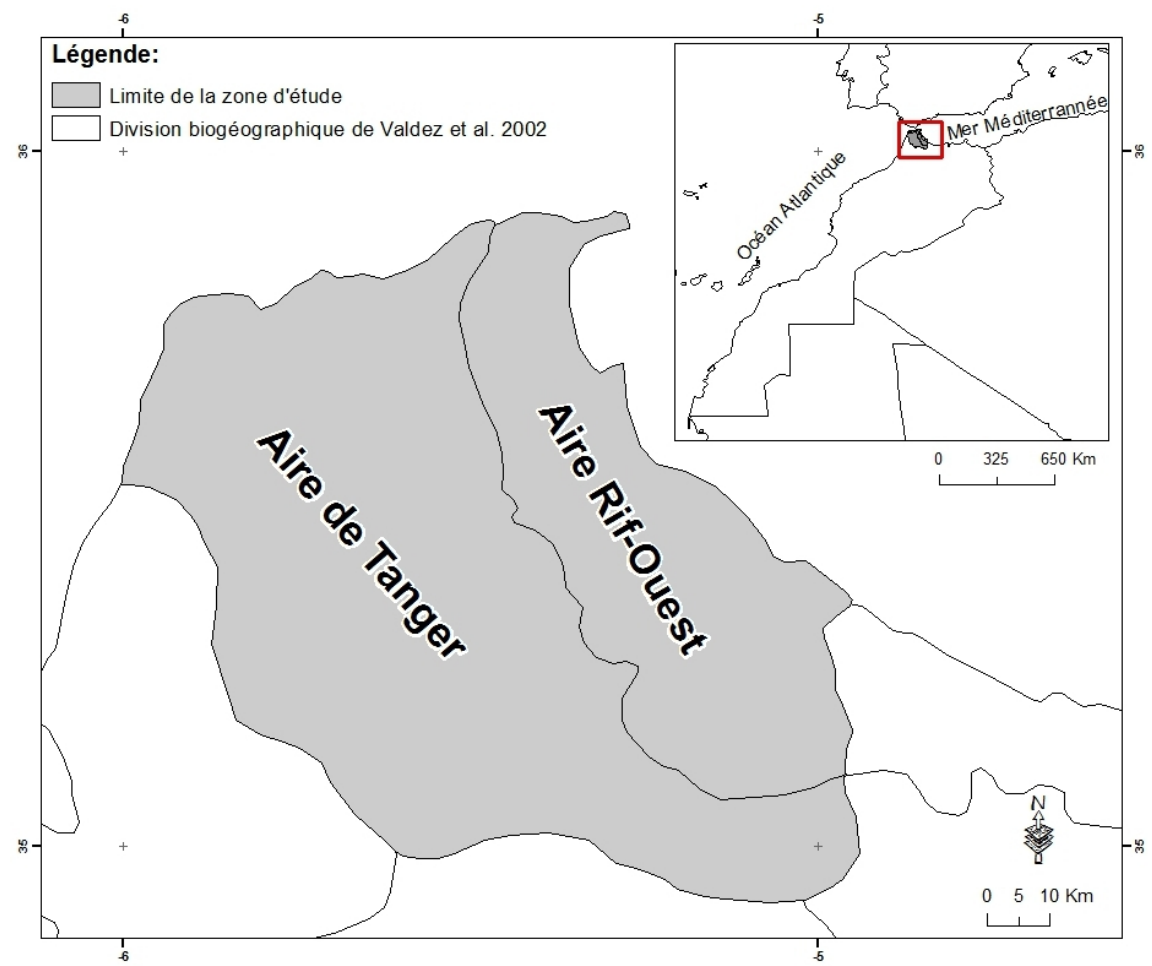

Fig.1. Aire d'étude ; 1 : Tanger ; 2: Rif Ouest. 


\section{2) Méthodologie}

\section{Inventaire général de la richesse floristique}

Le point de départ de cette étude est la mise en place d'une base de données contenant l'inventaire complet des espèces, sous-espèces et variétés existantes dans notre aire d'étude, avec leurs aires biogéographiques et leurs statuts de conservation (rares ou très rares, à contrôler et à protéger, vulnérables et endémiques). Les documents de base exploités sont :

- le Catalogue des Plantes vasculaires du Nord du Maroc (Valdès et $a l, 2002)$ pour tout ce qui concerne la nomenclature taxonomique et la distribution biogéographique ;

- le Catalogue des Plantes Vasculaires rares, menacées ou endémiques du Maroc (Fennane et Ibn Tatou, 1998) pour tout ce qui a trait aux différents statuts de rareté, de menace et d'endémisme ;

- l'article sur les Relevés floristiques et catalogue des plantes médicinales dans le Rif occidental (Benabid et Bellakhdar, 1987), qui a été exploité aussi pour ce qui concerne les espèces menacées.

Il est à signaler que les auteurs précités donnent des significations concrètes aux termes rare, très rare, à contrôler, à protéger et vulnérable. Ces significations sont reprises ci-dessous avec les symboles utilisés pour chaque catégorie d'espèces :

- RR : très rare (nombre de localités inférieur ou égal à 5);

- $\mathrm{R}$ : rare (en général signalé dans 1 ou 2 divisions du Catalogue des plantes du Maroc de Jahandiez et Maire (1931-34) et Emberger et Maire (1941));

- $\quad$ RR ?, R ? : Soupçonné être rare ou très rare;

- ? : taxon éteint ou de présence douteuse;

- $\mathrm{C}$ : à contrôler (espèce végétale dont l'exploitation éventuelle devrait être contrôlée);

- $\quad \mathrm{P}$ : à protéger (espèce rare ou en voie de disparition);

- V : vulnérable (en voie de régression et pourrait devenir rare à court terme).

Dans ce travail, les quatre premiers statuts dus à Fennane et Ibn Tatou (1998) sont adoptés en considérant, dans la catégorie "soupçonné rare ou très rare", tous les taxons estimés éteints par Fennane et Ibn Tatou (1998) mais signalés présents dans plus qu'une division du Catalogue des plantes vasculaires du Nord du Maroc de Valdés et al. (2002). Les taxons n'appartenant pas à ces catégories et qui sont signalés dans les trois derniers statuts (espèces à contrôler, à protéger et vulnérables) proposés par Benabid et Bellakhdar (1987), sont regroupés sous le terme de taxons menacés. 


\section{Inventaire des PAM}

A partir de la base de données de la flore totale, on a procédé à l'identification de l'ensemble des espèces et taxons infra-spécifiques considérés comme Plantes Aromatiques et Médicinales, en précisant leur usage et exploitation. Pour ce faire, on s'est référé aux études ethnobotaniques réalisées au Nord du Maroc (Benabid et Bellakhdar, 1987; Benabid, 1998; Dam, 2004; El Ouahhabi, 2007) et à l'échelle de l'ensemble du territoire national (Hmamouchi, 1999; Bellakhdar, 1997), comme à d'autres références bibliographiques internationales (Gonzalez-Tejero et al., 2007; Lubbe and Verpoorte, 2011) et bases de données accessibles sur le web telles que Plants For A Future (www.pfaf.org), Phytomania (www.phytomania.com) et UICN-Med (www.uicnmed.org/ nabp/database/ NA_Plant.htm).

\section{Analyse phytosociologique et écologique des PAM}

Dans le but d'évaluer la phytosociologie et l'écologie des PAM, 449 relevés floristiques réalisés, dans la zone d’étude, par Benabid (1984), Benabid et Bellakhdar (1987) et Quézel et al. (1992), ont été saisis. A partir de ces relevés, les estimations d'abondance-dominance de l'échelle de Braun-Blanquet sont transformées en pourcentages (\%) comme suit : $+=1 \% ; 1=3 \% ; 2=15 \% ; 3=37,5 \% ; 4$ = 62,5\%; 5 = 87,5\%.

Une matrice Espèces x Relevés est alors constituée comprenant le pourcentage (\%) d'abondance-dominance de chacune des espèces. Cette matrice a été soumise à une analyse de classification, après transformation logarithmique, en utilisant la méthode de variance minimale.

Par ailleurs, une autre matrice contenant les données de sept variables environnementales (altitude(en mètres), orientation, pente (en degrés), substrat géologique, type de sol, bioclimat et type d'hiver) des mêmes relevés a été utilisée pour la caractérisation environnementale des groupements issus de l'analyse de classification. L'analyse de corrélation canonique (ACC) est employée ici pour établir des relations statistiques entre les deux matrices de données.

\section{RESULTATS ET DISCUSSION \\ 1) Inventaire de la flore Tingitane \\ Richesse floristique}

L’inventaire floristique de l'aire d'étude (Ouest Rif et Tanger) nous a permis de lister 2053 taxons appartenant à 145 familles. Les Angiospermes Dicotylédones et Monocotylédones représentent plus de 97,5\% de la richesse floristique totale avec 1571 et 431 taxons respectivement, alors que les Ptéridophytes et les Gymnospermes ne contiennent que 36 et 15 taxons, représentant ainsi $1,75 \%$ et $0,73 \%$ respectivement. 
Les familles des Papilionaceae (248 taxons), Asteraceae (232 taxons), Poaceae (204 taxons), Caryophyllaceae (108 taxons), Lamiaceae (82 taxons), Apiaceae (80 taxons), Scrophulariaceae (79 taxons) et Brassicaceae (78 taxons) regroupent à elles seules 1111 espèces et taxons infra-spécifiques, représentant plus de $54 \%$ de la richesse totale. Ces familles importantes au niveau de la Péninsule Tingitane occupent le même ordre d'importance parmi la richesse floristique marocaine totale (Fennane, 2004).

\section{- $\quad$ Rareté et endémisme}

La Péninsule Tingitane contient au moins 477 taxons importants pour la conservation. Parmi ces taxons, 276 espèces et sous-espèces sont rares ou très rares, soit $13,4 \%$ de la flore totale. A ces taxons rares, on doit ajouter 58 taxons soupçonnés être rares ou très rares. Dans la catégorie des taxons menacés, on compte au total 44 taxons (figure 2).

Du point de vue endémisme, on a 166 taxons endémiques marocains, 45 endémiques maroco-ibériques, 12 endémiques maroco-algériennes, 20 taxons endémiques des trois régions et une endémique du Maroc, Espagne et Iles Canaries. L'effectif total des endémiques s'élève donc à 244 taxons, soit un taux d'environ $12 \%$ de la flore totale.

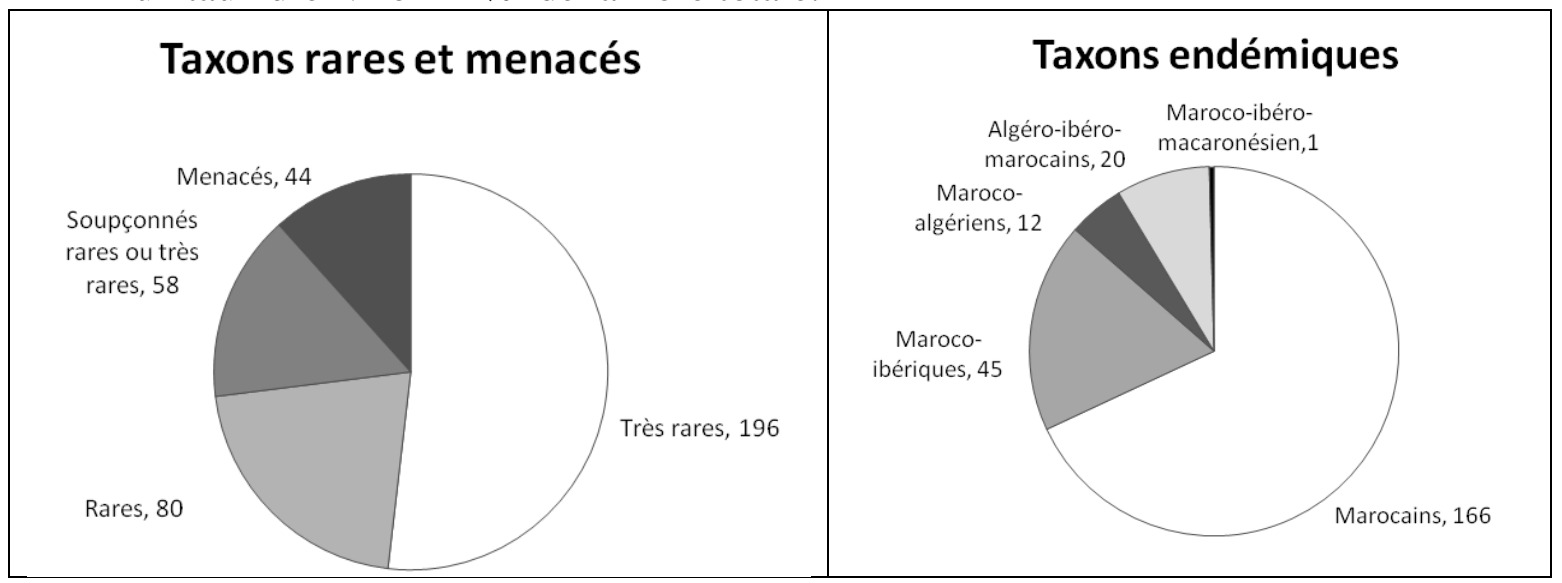

Fig.2. Répartition des espèces à statuts importants pour la conservation.

Il faut noter que certains taxons peuvent appartenir à la fois aux catégories de rareté, d'endémisme et parfois même aux espèces menacées. Aussi faut-il indiquer qu'en gardant seulement les taxons rares et très rares, ceux qui sont menacés et ceux endémiques exclusifs du Maroc (sans compter les taxons éteints et soupçonnés, ni ceux endémiques du Maroc et de la Péninsule ibérique ni ceux endémiques du Maroc et de l'Algérie), il faut compter globalement 471 taxons, soit un taux d'environ $23 \%$ qui doivent faire l'objet de conservation. 


\section{2) Les PAM de la Péninsule Tingitane}

\section{Richesse en PAM}

Les PAM sont représentées par 630 taxons dans la Péninsule Tingitane, ce qui constitue presque $31 \%$ de la richesse floristique totale du territoire étudié. La moitié de ces PAM appartiennent aux Familles des Asteraceae (59 taxons), Papilionaceae (51 taxons), Lamiaceae (43 taxons), Apiaceae (30 taxons), Brassicaceae (22 taxons), Euphorbiaceae (21 taxons), Rosaceae (21 taxons), Caryophyllaceae (17 taxons), Rununculaceae (16 taxons), Polygonaceae (14 taxons) et Poaceae (27 taxons).

\section{- $\quad$ Rareté et endémisme des PAM}

La rareté concerne 77 taxons, soit environ 12,2\% de l'ensemble des PAM de la Péninsule Tingitane, dont 45 espèces et sous-espèces très rares, 18 rares, 14 soupçonnées être rares (figure 3). Le nombre de PAM considérées menacées s'élève à 22 taxons.

De point de vue endémisme, 18 espèces et sous-espèces sont endémiques exclusives du Maroc. Ce chiffre augmente à 25 si on y ajoute 4 autres taxons endémiques du Maroc et de l'Algérie et 3 du Maroc et de la péninsule ibérique (figure 3).

Au total, la Péninsule Tingitane abrite 106 taxons de PAM appartenant à différentes catégories de rareté et d'endémisme. Ces taxons exigent des dispositions réglementaires particulières pour assurer leur conservation (Annexe 1). Il est important de signaler ici, que parmi les 18 endémiques marocaines de la liste précitée, 5 taxons sont très rares (Anthyllis vulneraria subsp. fatmae, Astragalus fontianus, Stachys fontqueri, Stachys officinalis subsp. algeriensis, Poa pratensis subsp. atlantis) et 2 sont rares (Abies marocana, Origanum grosii).

\section{Taxons rares et menacés}

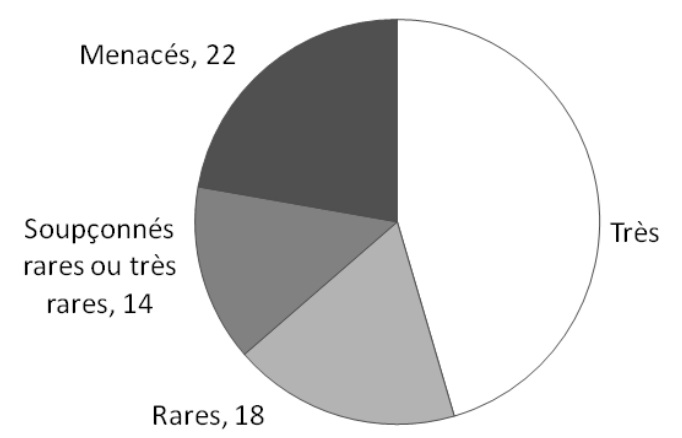

\section{Taxons endémiques}

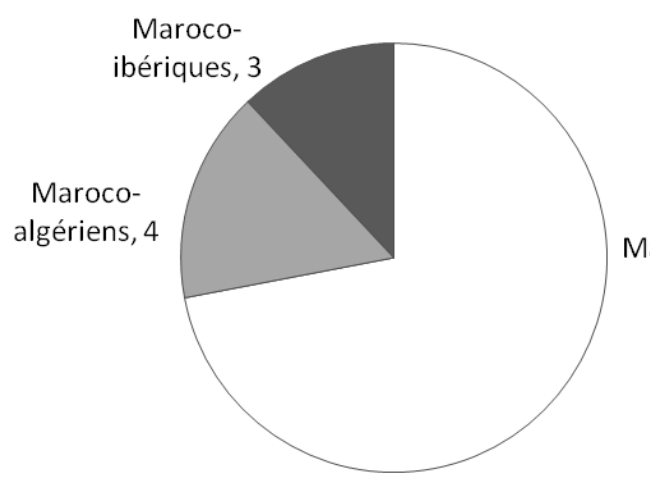

Fig. 3. Effectifs des PAM rares, menacées et endémiques. 
- $\quad$ Les PAM exploitées :

Parmi les 630 Plantes Aromatiques et Médicinales recensées dans l'aire d'étude, 23 seulement sont largement exploitées pour divers usages ethnobotaniques (Dam, 2004; DREF du Rif-MEDA, 2004; El Ouahhabi, 2007; APDN, 2012): Pistacia lentiscus, Dittrichia graveolens, Dittrichia viscosa, Inula montana, Tanacetum annuum,Ceratonia siliqua, Cistus ladanifer subsp. mauritianus, Cistus laurifolius subsp. atlanticus, Tetraclinis articulata, Arbutus unedo, Lavandula dentata var. dentata, Lavandula stoechas subsp. stoechas, Mentha pulegium, Origanum compactum, Origanum grosii, Rosmarinus officinalis var.officinalis, Satureja nepeta subsp. nepeta, Thymbra capitata, Thymus Willdenowii, Lauru snobilis, Myrtus communis, Ononis natrix subsp. Natrix et Pinus halepensis. Elles ne représentent que 3,7\% du grand potentiel de PAM dont recèle la région. Ceci est dû, comme signalé dans la Stratégie Nationale de Développement des PAM au Maroc (HCEFLCD, 2008), au manque de connaissances sur leur utilité ou à l'absence de valorisation et de débouchés commerciales.

A signaler aussi que parmi les 106 taxons de la liste établie et proposée en annexe 1, des espèces telles que Thymbra capitata, Laurus nobilis, Tanacetum annum et Calluna vulgaris, sont très fortement menacées d'extinction en raison de leur grande exposition aussi bien à la pression anthropozoogène (espèces largement exploitées) qu'aux problèmes d'incendies qui sont devenus plus fréquents et plus étendus suite à la conjugaison de conditions atmosphériques défavorables et une utilisation irrationnelle des terres (Fikri et al, 2004).

\section{- $\quad$ Usages des PAM}

L’usage des PAM recensées dans la Péninsule Tingitane touche des domaines très variés. On note à ce propos celles utilisées en phytothérapie moderne, en pharmacopée locale traditionnelle, en exploitation industrielle comme bois d'œuvre, liège et goudrons, en artisanat pour la fabrication de petits objets domestiques, outillages et bois de feu, en alimentation pour assaisonnement des plats ou comme épices et en utilisations cosmétiques et hygiéniques. D’autres espèces de PAM sont utilisées comme aromates dans les boissons et autres, pour les soins vétérinaires, en tant que plantes fourragères et à utilisation pastorale, pour les rites funéraires, en sorcellerie et magie ou comme drogue. Des espèces de PAM sont butinées par les abeilles, et sont de ce fait, des plantes mellifères alors que d'autres sont toxiques. Le tableau 1 illustre ces différentes utilisations. 
Tableau 1.Usages des PAM.

\begin{tabular}{ll}
\hline Types d'usages & Nombre d'espèces \\
\hline Usage en Médecine traditionnelle & 330 \\
Usage en Médecine moderne & 308 \\
Usages Alimentaires, Epices, Additifs & 127 \\
Usage Ornemental & 93 \\
Toxique & 70 \\
Usages Pastoraux et fourragers & 59 \\
Usages Artisanaux (vannerie, tannerie, menuiserie...) & 50 \\
Usages Aromatiques, boissons, condimentaires & 48 \\
Usages cosmétiques et hygiéniques & 46 \\
Usages Industriels & 29 \\
Mellifère & 28 \\
Usages Vétérinaires & 17 \\
Usage en sorcellerie, magie et encens & 10 \\
Drogue & 8 \\
\hline
\end{tabular}

Il faut noter que plusieurs taxons sont à utilisation multiple. Néanmoins, dans le lot des 308 PAM recensées en phytothérapie moderne, 109 sont exclusives pour cet usage. Ceci nous amène à dire que des opportunités réelles de développement pour au moins une partie de ces espèces existent. Les centres de recherche et de développement sont, de ce fait, interpelés pour cette mise en valeur. Aussi, des efforts doivent être consentis en termes de promotion et de valorisation notamment pour les PAM d'industrie cosmétique et alimentaire.

\section{3) Les PAM dans les groupements végétaux naturels Classification des groupements}

La matrice analysée comprend 449 relevés floristiques, englobant 601 espèces. Le dendrogramme de classification numérique a permis de reconnaître 9 groupements végétaux majeurs qui sont dégagés au même seuil de similitude (distance euclidienne égale à 500). Ces groupements, dont les descriptions ci-dessous ont fait référence aux nomenclatures des associations telles quelles sont définies par Benabid (1984), sont les suivants (figure 4) :

1. les cocciféraies (37 relevés) : Elles sont marquées par l'abondance et la constance de Quercus coccifera, Pistacia lentiscus, Smilax aspera et Phylleria latifolia. La quasi-totalité des relevés de ce groupement se rapporte à l'association Rusco-Quercetum cocciferae. La richesse spécifique totale dans ce groupement s'élève à 140 espèces.

2. les oléastraies (41 relevés) : Ce groupement est surtout dominé par Olea europaea var. sylvestris de l'association Tamo-Oleetum sylvestris (30 relevés) et comprend aussi des relevés de frênaies et certaines subéraies caractérisées par l'absence d'Arbutus unedo et Erica arborea et par l'importance de certaines lianes telles que 
Smilax aspera var altissima, et Aristolochia baetica. Le nombre total d'espèces de ce groupement est égal à 120.

3. les formations thermophiles (110 relevés): Ce groupement comprend des relevés de formations forestières ou matorrals caractérisés par l'omniprésence du lentisque. Environ la moitié des relevés de ce groupement correspond à des forêts dominées par Tetraclinis articulata (Calycotomo-Tetraclinetum articulatae). On y rencontre aussi des forêts dominées par Quercus rotundifolia et Pinus halepensis. Les matorrals inclus dans ce groupement sont surtout structurés par Quercus coccifera, Buxus balearica, Pistacia lentiscus et Phillyrea latifolia. La richesse de ce groupement atteint 249 espèces.

4. les subéraies (49 relevés) : Ce groupement renferme les relevés des deux associations des subéraies de la zone d'étude (MyrtoQuercetum suberis et Teucrio-Quercetum suberis) et parfois des mélanges de chênes liège et zène (Cytiso-Quercetum canariense) auxquels s'ajoutent des matorrals dominés par Erica arborea et Arbutus unedo. L'échantillonnage des subéraies comprend 181 espèces.

5. la pinède maritime (40 relevés) : Ce groupement est dominé par des peuplements de Pinus pinaster contenant les relevés des deux faciès à $P$. pinaster var. iberica et à $P$. pinaster var. maghrebiana différenciés par Benabid (1984). En plus, ce groupement intègre aussi certaines chênaies vertes de l'association Smilaci-Quercetum rotundifoliae. Les pinèdes englobent 216 espèces et s'avèrent très riches compte tenu du nombre de relevés considérés.

6. les forêts caducifoliées (32 relevés): Formations forestières dominées par Quercus pyrenaica et/ou Quercus canariensisse rapportant essentiellement aux associations Cytiso-Quercetum canariense et Violo-Quercetum pyrenaicae. La richesse floristique des forêts caducifoliées atteint 163 espèces.

7. les matorrals (60 relevés): Ce groupement est représenté par différents types de formations basses. Il s'agit généralement de cistaies mais on y trouve aussi d'autres types de matorrals tels que la chênaie naine à Quercus lusitanica. Les matorrals renferment 127 espèces et représentent le groupement le moins riche par rapport à la taille de l'échantillonnage.

8. les formations montagnardes (57 relevés) : Ce groupement comprend des relevés qui sont dominés par différentes essences arboricoles caractéristiques des hautes altitudes (Paeonio-Quercetum rotundifoliae, Berberido-Cedretum atlanticae, Paeonio-Abietetum maroccanae sous-association cedretosum, Trifolio-Quercetum 
fagineae, Polysticho-Prunetum lusitanicae). Certaines garrigues et matorrals de montagne appartiennent aussi à ce groupement. Ce groupement est aussi très riche que les pinèdes maritimes puisqu'il contient 306 espèces.

9. la sapinière (23 relevés) : Il s'agit de relevés de sapinière sans cèdre et dont Paeonia maroccana est bien représentée (Paeonio-Abietetum maroccanae). Les deux sous associations abietetosum et quercetosum fagineae sont toutes les deux représentées dans ce groupement. En dépit du nombre réduit de relevés, la richesse spécifique dans ce groupement s'élève à 100 espèces.

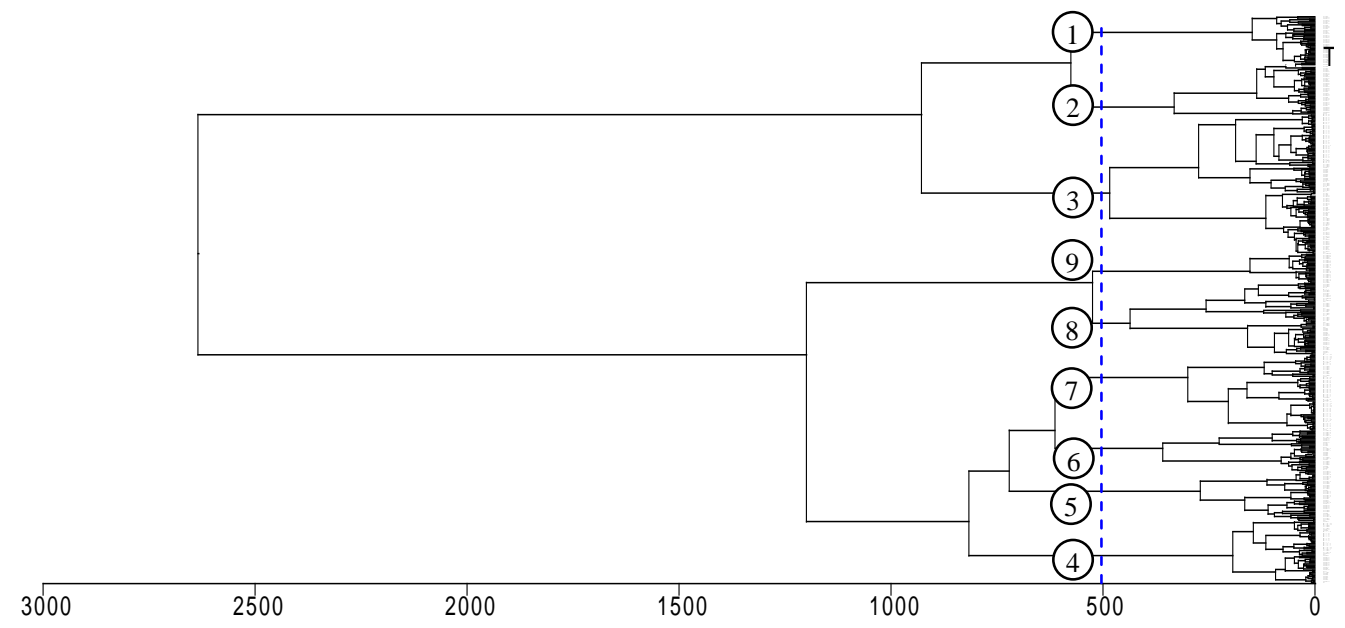

Fig.4. Dendrogramme de classification des groupements végétaux.

Ces groupements se répartissent en fonction des grandes variables environnementales régionales. L'analyse de corrélation canonique résume ces variables en deux axes : le premier est climatique et le second édaphique (Fig. 5). En effet, les groupements montagneux et la sapinière se développent en haute altitude sur substrats calcaires dolomitiques en bioclimat perhumide sous sa variante froide. De leur côté, les forêts caducifoliées constituent un groupement calcifuge et prospèrent en bioclimats humide à perhumide frais. A l'opposé, les formations thermophiles, l'oléastraie et la cocciféraie préfèrent des bioclimats subhumide et semi-aride sous variantes tempérée à chaude. Les matorrals et les subéraies préfèrent les substrats gréseux alors que les pinèdes se développent préférentiellement sur calcaires dolomitiques. 


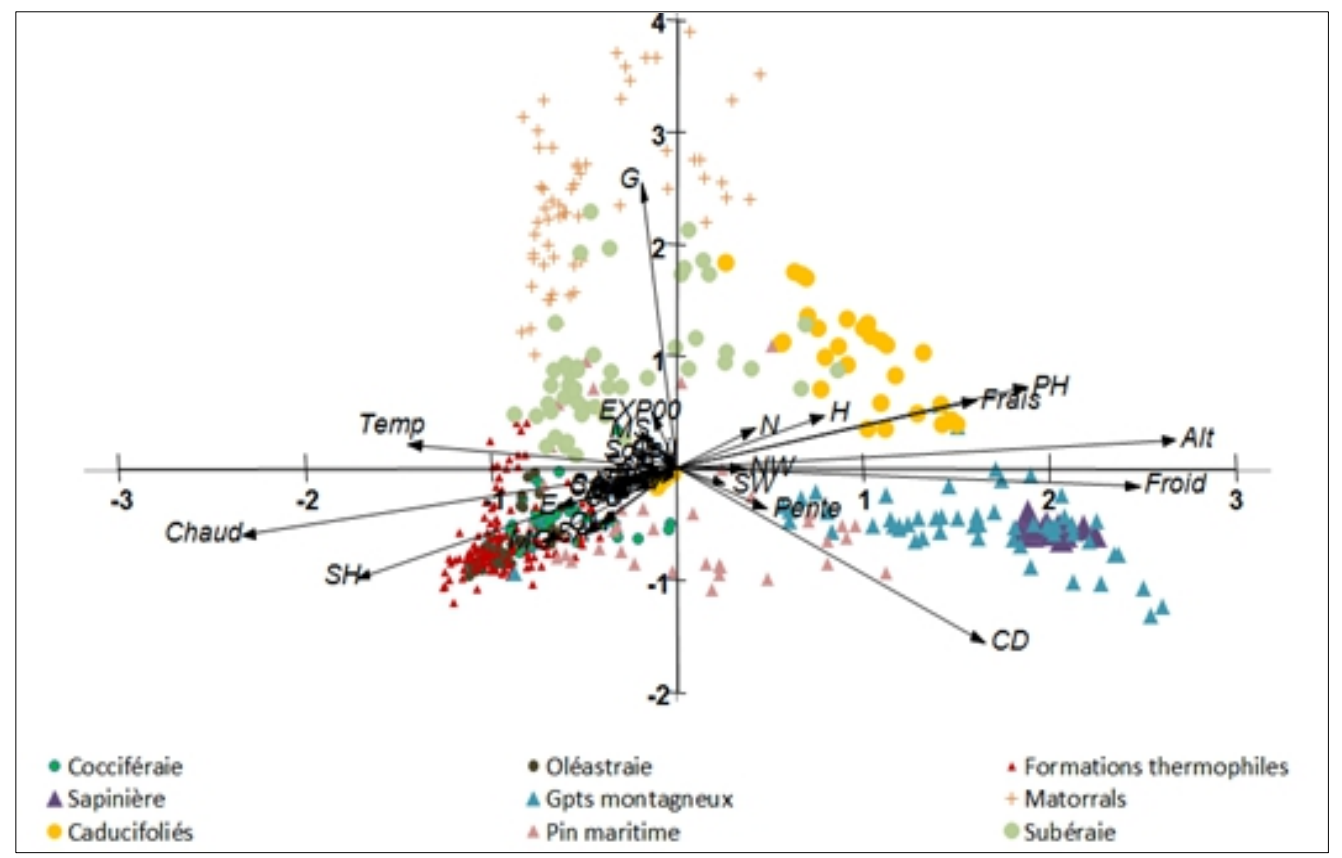

Fig. 5. Analyse de Corrélation Canonique (ACC) des groupements végétaux avec les variables environnementales

\section{- $\quad$ Richesse, rareté et endémisme des PAM dans les groupements}

Les groupements décrits ci-dessus sont riches en PAM avec des pourcentages allant de 29 à $48 \%$ de leur richesse totale. La richesse totale de ces groupements en PAM est égale à 171 taxons. La grande majorité des PAM est commune à plusieurs groupements mais34 taxons sont rares ou menacés et 5 sont des endémiques marocaines.

Le groupement des formations montagnardes se distingue par les valeurs les plus élevées en PAM exclusives, endémiques, rares et menacées (Tabl. 2). Les pinèdes maritimes contiennent aussi un nombre important d'espèces rares ou menacées. Quant aux forêts caducifoliées et à la sapinière, elles contiennent relativement moins de taxons rares ou menacés mais dont la proportion par rapport aux richesses respectives en PAM est assez importante: 15 et $17,2 \%$.

A l'opposé, les groupements des forêts de basses altitudes, y compris les subéraies, ont des pourcentages de richesse en PAM relativement supérieurs mais avec de faibles pourcentages de rareté et d'endémisme. En fait, les oléastraies et les cocciféraies, ainsi que les matorrals, contiennent peu de PAM rares et menacées; 6,9\%, 9,2\%et 10,2\% respectivement. Ils n’hébergent aucun taxon endémique. Les PAM des formations thermophiles contiennent $11,3 \%$ de taxons rares et menacées et une seule endémique marocaine (Origanum grosii). Le pourcentage des taxons rares et menacés des subéraies, en grande partie localisées à basse altitude mais qui peuvent 
s'étendre jusqu'à environ 1600 m d'altitude, représente 12,2\% des PAM.

Il s'avère donc que, malgré l'abondance relative des PAM dans les groupements de basse altitude, les différents groupements de montagne (sapinière, cédraies, pinèdes maritimes, forêts caducifoliées, chênaies vertes) se caractérisent par une proportion relativement plus importante de PAM exclusives, endémiques, rares ou menacées. En particulier, le groupement des formations montagnardes, tel que définit plus haut, peut être considéré comme un véritable refuge pour certains taxons de PAM endémiques, rares, menacées et exclusives. Ces dernières sont : Astragalus armatus ssp numidicus, Astragalus fontianus, Atropa baetica, Ephedra nebrodensis, Erodium cicutarium, Prunella vulgaris, Prunus avium, Sedum acre, Sedum album, Sorbus aria, Sorbus torminalis, Taxus baccata, Vicia sativa, Viola riviniana.

Tableau 2. Richesse totale, rareté et endémisme des groupements.

\begin{tabular}{llllllll}
\hline & $\begin{array}{l}\text { Nombre } \\
\text { de } \\
\text { relevés }\end{array}$ & $\begin{array}{l}\text { Richesse } \\
\text { totale }\end{array}$ & $\begin{array}{l}\text { Nombre } \\
\text { de } \\
\text { taxons } \\
\text { PAM }\end{array}$ & $\begin{array}{l}\text { \% en } \\
\text { PAM }\end{array}$ & $\begin{array}{l}\text { PAM } \\
\text { exclusives }\end{array}$ & $\begin{array}{l}\text { PAM } \\
\text { Rares et et } \\
\text { menacées }\end{array}$ & $\begin{array}{l}\text { PAM } \\
\text { Endémiques } \\
\text { marocaines }\end{array}$ \\
\hline Cocciféraies & 37 & 140 & 65 & 46 & 1 & 6 & 0 \\
\hline Oléastraies & 41 & 120 & 58 & 48 & 4 & 4 & 0 \\
\hline $\begin{array}{l}\text { Formations } \\
\text { thermophiles }\end{array}$ & 110 & 249 & 97 & 39 & 12 & 11 & 1 \\
\hline Subéraies & 49 & 181 & 74 & 41 & 4 & 9 & 1 \\
\hline Pinèdes maritimes & 40 & 216 & 83 & 38 & 3 & 14 & 3 \\
\hline Forêtscaducifloliées & 32 & 163 & 60 & 37 & 3 & 9 & 1 \\
\hline Matorrals & 60 & 127 & 39 & 31 & 1 & 4 & 0 \\
\hline $\begin{array}{l}\text { Formations } \\
\text { montagnardes }\end{array}$ & 57 & 306 & 92 & 30 & 14 & 20 & 5 \\
Sapinière & 23 & 100 & 29 & 29 & 0 & 5 & 1 \\
\hline
\end{tabular}

\section{CONCLUSION}

L'aire d'étude, d'une superficie représentant moins de $1 \%$ du territoire national, abrite presque 50\% de la flore marocaine. Parmi 2053 espèces et taxons infra-spécifiques rencontrés dans la Péninsule Tingitane, 8\% des taxons sont endémiques du Maroc, 13,4\% sont des taxons rares à très rares et 2,4\% des espèces ou taxons infra-spécifiques sont menacés. Ce patrimoine englobe 630 taxons de Plantes Aromatique et Médicinales qui méritent d'être à la fois conservées et valorisées.

En effet, 17\% de ce patrimoine (106 taxons) est constitué de taxons soit rares ou très rares et/ou menacés et/ou endémiques du Maroc, d'Algérie et de la péninsule ibérique, qui doivent être prioritaires dans tout programme de conservation. De même, à peine 3,7\% de la grande richesse des PAM de la région est exploitée alors qu'il existe un grand potentiel d'usages multiples à promouvoir et à valoriser. Les services de recherche et de développement 
sont, dans ce sens, interpelés pour inscrire d'urgence la conservation, la promotion et la valorisation de ce patrimoine dans leurs programmes d'action.

L'analyse des principales études phytosociologiques et écologiques disponibles indique que seulement une faible proportion, représentant moins du tiers des PAM de la région, se développe dans les forêts et les matorrals naturels; la grande partie du patrimoine en PAM est cantonnée dans des milieux marginaux ou très dégradés. Parmi les neuf groupements caractérisés du point de vue floristique, climatique et édaphique, les PAM représentent presque la moitié de la richesse des forêts naturelles de basse altitude. Les groupements de haute altitude hébergent des proportions relativement importantes de PAM exclusives, endémiques, rares et menacées.

\section{References:}

Agence pour la promotion et le développement du Nord (APDN), 2012. Etude sur le secteur des Plantes Aromatiques et Médicinales dans la Réserve de Biosphère Intercontinentale de la Méditerranée. Etude des potentialités en PAM et des possibilités de valorisation dans la zone de la RBIM du Maroc, Mission 1.

Bellakhdar J., 1997. Contribution à l'étude de la pharmacopée traditionnelle au Maroc : La situation actuelle, les produits, les sources du savoir. Enquête ethnobotanique de terrain réalisée de 1969 à 1992. Thèse, Université de Metz, 2 volumes, 1157p.

Bellakhdar J., 2006. Plantes médicinales au Maghreb et soins de base (Précis de phytothérapie moderne). Editions Le Fennec, Casablanca (Maroc), Avril 2006, 386p.

Benabid A., 1984. Etude phytoécologique des peuplements forestiers et préforestiers du Rif Centro- Occidental (Maroc). Travaux de l'Institut Scientifique, Série Botanique, 34: 64p.

Benabid A., 1998. Flore et végétation du parc national de Talassemtane, Chefchaouen - Maroc. Programme MEDA, Projet de développement participatif des zones forestières et périforestières de la province de Chefchaouen, 159p.

Benabid A., 2002. Le Rif et le Moyen-Atlas (Maroc): Biodiversité, menaces, préservation. African Mountains High Summit Conference Nairobi, Kenya, 6-10 mai 2002.

Benabid A. et Bellakhdar J., 1987. Relevés floristiques et catalogue des plantes médicinales dans le Rif occidental (Missions ethnobotaniques 19841987). Al Biruniya 3(2): 87-120.

Dam A., 2004. Contribution à l'étude de la filière des Plantes Aromatiques et Médicinales dans le Rif central et occidental du Maroc. Concours général de 
la formation professionnelle agricole, $16^{\text {ème }}$ édition, ITSA de Tétouan, Juillet 2004, 72p.

El Ouahhabi M.Y., 2007. Mise en place d'un Arboretum Aromatique de préservation et de référence des Plantes Aromatiques et Médicinales de la région du Nord. Concours général de la formation professionnelle agricole, $19^{\text {eme }}$ édition, ITSA de Tétouan, Juillet 2007, 82p.

El Oualidi J., H. Khamar, M. Fennane, M. Ibn Tattou, S. Chauvet, M. Sghir Taleb, 2012. Checklist des endémiques et spécimens types de la flore vasculaire de l'Afrique du Nord. Document de l'Institut Scientifique, $\mathrm{N}^{\circ} 25$, 2012.

Emberger L. \& R. Maire 1941. Catalogue des Plantes du Maroc. Volume IV. Minerva, Alger.

Fennane M., 2004. Propositions de zones importantes pour les plantes au Maroc (ZIP). Atelier national ' 'zones importantes pour les plantes au Maroc’' Rabat 11-12/10/2004.

Fennane M. et M. Ibn Tatou, 1998. Catalogue des plantes vasculaires rares, menacées ou endémiques du Maroc. Bocconia 8 (Monographie), 279p.

Fennane M., \& M. Ibn Tattou, 1999. Observations sur la flore vasculaire endémique, rare ou menacée du Maroc. Flora Mediterranea 9 - 1999.

Fikri K.B, Ismaili M., Fikri S.B., Tribak A., 2004. Problèmes de dégradation de l'environnement par la désertification et la déforestation : impact du phénomène au Maroc. Sécheresse n 4, vol. 15 : 307-20, décembre 2004.

Gonzalez-Tejero M.R., M. Casares-Porcel, C.P. Sanchez-Rojas, J.M. Ramiro-Gutierrez, J. Molero-Mesa, A. Pieroni, M.E. Giusti, E. Censorii, C. de Pasquale, A. Della, D. Paraskeva-Hadijchambi, A. Hadjichambis, Z. Houmani, M. El-Demerdash, M. El-Zayat, M. Hmamouchi, S. ElJohrig, 2007. Medicinal plants in the Mediterranean area: Synthesis of the results of the project Rubia. Journal of Ethnopharmacology 116: 341-357.

Haut-Commissariat aux Eaux et Forêts et à la Lutte Contre la Désertification (HCEFLCD), 2008. Stratégie nationale de développement du secteur des plantes aromatiques et médicinales. Mission USAID/Maroc, 72p.

Hmamouchi M., 1999. Les plantes médicinales et aromatiques marocaines : utilisation, biologie, écologie, chimie, pharmacologie, toxicologie, lexiques. Imprimerie de Fédala, Mohammedia, 389p.

Hurtel J.M., 2001. Phytothérapie, plantes médicinales, aromathérapie, huiles essentielles: www.phytomania.com . Consulté le (11/05/2013).

Jahandiez R. et R. Maire 1931-34. Catalogue des plantes du Maroc, 3 vol. Ed. Minerva, Alger.

Lubbe A. and R. Verpoorte, 2011. Cultivation of medicinal and aromatic plants for specialty industrial materials. Industrial Crops and Products 34(1): 785-801. 
Medail, F. \& P., Quézel, 1997. Hot-Spots Analysis for Conservation of Plant Biodiversity in the Mediterranean Basin.Annals of the Missouri Botanical Garden 84 (1): 112-127.

Quézel P., Barbero M. Benabid A., Loisel R. \& Rivas-Martinez S., 1992.Contribution à l'étude des groupements préforestiers et des matorrals rifains. Ecol. Médit. 14(1/2): 57-122.

Valdès B., 1991. Andalucia and the Rif. Floristic links and a common flora. Botanica Chronika 10, 117-124.

Valdès B., M. Rejdali, A. Achhal EI Kadmiri, J.L. Jury, J.M. Montserrat (Editeurs), 2002. Catalogue des Plantes Vasculaires du Nord du Maroc. Vol. 1 \& 2. CSIC. Madrid.Annexe 1 : Liste des taxons PAM très rares, rares, menacés et endémiques dans la Péninsule Tingitane (Maroc). RR : très rare ; $\mathrm{R}$ : rare ; $\mathrm{R}$ ? : soupçonné être rare ; $\mathrm{C}$ : à contrôler ; $\mathrm{P}$ : à protéger ; $\mathrm{V}$ : vulnérable; $\mathrm{E}$ : endémique du Maroc; A : endémique du Maroc et de l’Algérie ; I : Endémique du Maroc et de la péninsule Ibérique.

\section{Alliaceae}

C 1- Allium triquetrum L.

Apiaceae (Umbelliferae)

RR 2- Daucus carota Subsp. Carota (L.) Thell.

R 3- Daucus carota Subsp. Gummifer Hook.f (=D. carota subsp. fontanesii Thell. =D. carota subsp. hispanicus auct.)

RR 4- Oenanthe fistulosa L.

E 5- Peucedanum vogelianum Emb.et Maire (=P. officinale subsp.vogelianum (Emb.et Maire) Maire.)

C 6- Pimpinella tragium vill.

C 7- Sanicula europaea L.

RR 8- Torilis japonica (Houtt) DC (=T. anthriscus (L.) C.C.Gmel)

Aspleniaceae

R? $\quad$ 9-Asplenium scolopendrium Subsp. scolopendrium L

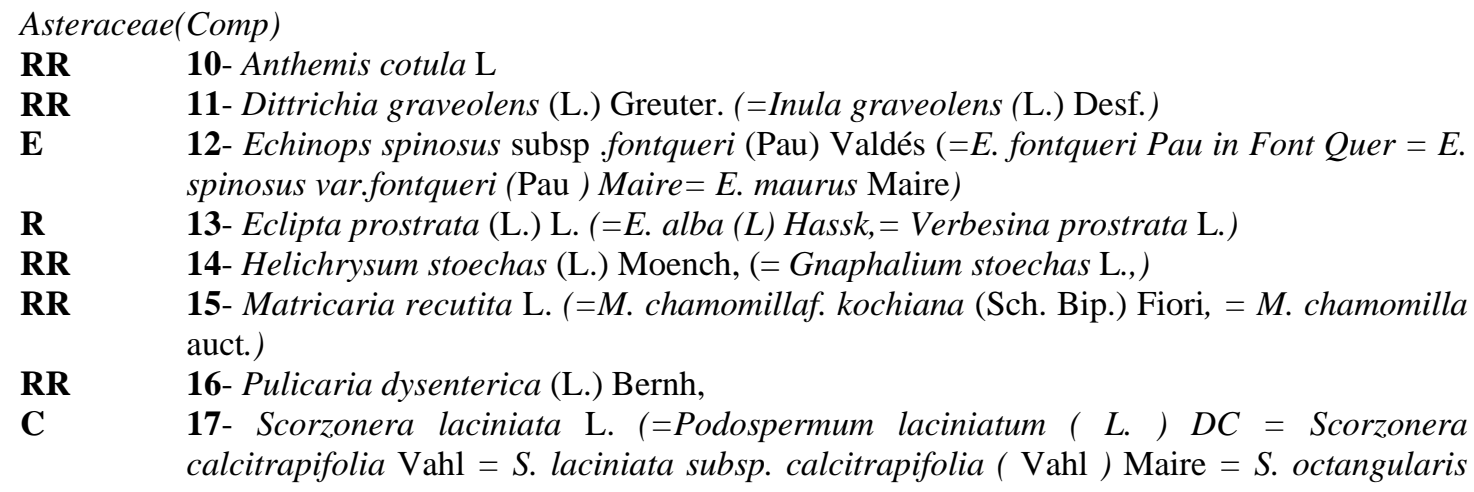


Wild)

RR 18- Senecio jacobaea L. (= S. foliosus Salzm. Ex DC.)

RR 19- Tanacetum annum L.

Betulaceae

R 20-Alnus glutinosa (L.) Gaertn.

Brassicaceae (Cruciferae)

RR 21- Raphanus raphanistrum subsp. landra (DC) Bonnier et Layens

Caprifoliaceae

C 22- Sambucuse bulus L.,

Caryophyllaceae

R 23- Herniaria hirsuta L.

Chenopodiaceae

R? 24-Salsola soda L.

Cistaceae

R? 25-Cistus creticus subsp. eriocephalus (vir.) Greuter et Burdet $(=C$. villosus L. $=C$. PauiSennen $=C$. Riphienenianus $\mathrm{Pau}=C$. Sennenianus Oau $=C$. Villosus var. mauritanicus (Viv) Grosser $=C$. vinyalsi Sennen)

E 26-Cistus laurifolius subsp. atlanticus (Pit.) Sennen et Mauricio

R? - I 27-Cistus populifolium subsp. major (Dunal) Hyrwood

Convallariaceae

R? 28- Polygonatum odoratum (Mill) Druce ( $=P$. officinale All. $=P$. vulgare Desf =Convallaria multiflora sensu Ball.)

\section{Cupressaceae}

V-E 29- Cupresus atlantica Gaussen.

RR 30 - Juniperus communis Subsp. hemisphaerica (C. Presl) Nyman.

Ericaceae

R 31-Calluna vulgaris (L.) Hull (=Erica vulgaris)

Euphorbiaceae

RR 32 - Euphorbia akenocarpa Guss.

RR 33- Euphorbia paniculata Subsp. paniculata

RR 34- Euphorbia segetalis L. (=E. humbertiana Maire = E. miricornis Maire et Wilczek $=E$. pinea L.)

Fagaceae

R 35- Quercus lusitanica Lam. (=Q. fruticosa Brot. = Q. humilis Lam.)

Gentianaceae

C 36- Blackstonia perfoliata Subsp. Perfoliata (=Chlora. perfoliata (L.) L)

P-E 37- Centaurium pulchellum Subsp. grandifrorum (Batt.) Maire, (=Centaurium candelabrum $\mathrm{H}$. Lindb.) 
Geraniaceae

$\begin{array}{ll}\mathbf{R R} & \text { 38- Geranium columbinum L. } \\ \mathbf{R R} & \text { 39- Geranium lanuginosum Lam. }\end{array}$

Grossulariaceae

C 40-Ribes uva-crispa L. (=R. glossularia L.)

Hypericaceae (Guttiferae)

C 41- Hypericum montanum L.

Juncaceae

RR 42- Juncus acutus subsp. leopoldii ( Parl ) Snogerup

Lamiaceae(Labiatae)

R? 43- Melissa offcinalis L.

RR 44- Mentha cervina L. (=Menthax poinetiana Sennen et Mamicia = Preslia cervina (L.) Fresen.)

R-E 45- Origanum grosii Pau et Font Quer

R-C 46- Phlomis purpurea subsp. caballeroi (Pau.) Rivas mort. (=Phlomis caballeroi Pau.)

C 47- Prunella vulgaris L.

E 48- Salvia mauretii Batt. (=S. maroccana Batt et Pit $=$ S. haematodes var. atlantica Pau. Et Font Quer)

RR 49- Stachys fontqueri Pau. (=S. mollis auct.)

RR-E 50- Stachys officinalis subsp. algeriensis (Noê) Franco. (=Batonica algeriensis Noé = B. algeriensis var tangerina $=B$. officinalis var. algeriensis = Stachys officinalis var. tangerina $=$ B. officinalis)

R 51- Thymbra capitata (L.) Cav. (=Coridothymus capitatus (L.) Rchb = Thymus capitatus (L.) Hoffmanns et Link)

Lauraceae

$\mathbf{R}$

52- Lauru snobilis L.

Numphaeaceae

RR 53- Nymphaea alba L.

Ophioglossaceae

V 54- Osmunda regalis var. plumieri (Tausch) Mild.

Paeoniaceae

C 55- Paeonia coriacea Boiss. (=P. corallina subsp.coriacea (Boiss.) Maire $=$ P. mascula subsp. Coriacea (Boiss.) Malag = P. mascula subsp.maroccana Pau et Font Quer).

Papaveraceae

RR 56- Chelidonium majus L.

Papilionaceae $=$ Fabaceae

RR-E 57- Anthyllis vulneraria Subsp. fatmae Font Quer

$\mathbf{R}$ 58-Astragalus depressus $\mathrm{L}$.

RR-E 59- Astragalus fontianus Maire (=A. nummunlarioides Pau.) 
E 60- Astragalus menselii Romo

RR 61- Astragalus monspessulanus L.

RR 62- Astragalus stella Gouan

R? 63-Calycotome villosa (Poir.) Link.

C $\quad 64$ - Coronilla valentina Subsp. glauca (L.) Batt.

R? 65- Dorycnopsis gerardi (L.) Boiss(=Anthyllis gerardi L.)

RR 66- Lotus angustissimus L.

E 67- Ononis natrix subsp. arganietorum (Maire) Sirj. ( $=O$. Arganietrum Maire $=O$ .Arganietrum var. Rifana Emb \& Maire $=O$. Inaequifolia var. Minoirifolia Pau in Fontquer)

RR $\quad \mathbf{6 8}$ - Spartium junceum L.

RR $\quad 69$ - Vicia sativa Subsp. marocarpa (Moris) Arcang. (=V. macrocarpa (Moris) Bertol)

Pinaceae

R-E $\quad$ 70- Abies marocana Trab. (=Abies pinsapo subsp. Maroccana $=$ A. tazoatana)

R-P 71- Pinus nigra Arnold. (=Pinus nigra Subsp. mauretanica (Maire et Peyerimh.) Hey wood $=P$. clusiana subsp. mauretanica (Maire et Peyrimh) Maire $=P$. pyrenaica subsp. mauretanica (Maire et Peyerimh) O. Swartz)

C $\quad 72$ - Pinus pinaster var. maghrebiana Villar. $(=P$. hamiltonii Ten. $=P$. mesogeensis Fieschi et Gaussen $=$ P. pinaster Subsp. Hamiltonii (Ten) Villar.)

Plantaginaceae

RR 73- Plantago major subsp. Intermedia (Gilib.) Lange (=P. intermedia Gilib.)

Poaceae(Gram)

RR 74- Avena fatua subsp. fatua $\mathrm{L}$.

RR 75- Catabrosa aquatica (L.) P. Beauv.

RR-E $\quad \mathbf{7 6}$ - Poa pratensis subsp. atlantis Maire

C 77- Stipa bromoides (L) Dorfl (=Achnatherum brommoides(L) P. Beauv = Agrostic bromoides L. = Aristella bromoides $(\mathrm{L})$ Bertol. = Lasiagrostis bromoides $(\mathrm{L})$ Nevski. = Stipa aristella L.)

Polygonaceae

R? 78- Polygonium arenastrum Boreau(=P. aequale Lindom)

$\mathbf{R} \quad$ 79- Rumex palustis Sm. (=R .maritimus auct.)

Polypodiaceae

RR $\quad \mathbf{8 0}$ - Polypodium cambricum L.

Primulaceae

E 81- Coris monspeliensis Subsp. maroccana (Murb.) Greuter et Burdet (=C. monspeliensis $f$. denticulata H.Lindb)

R? - A 82- Primula acaulis Subsp. atlantica (Maire et Wilezek) Greuter et Burdet (=P. vulgaris subsp. atlantica (Mairet et Wilezek) Greuter et Burdet)

Rhamnaceae

$\mathbf{R}$

83- Frangula alnus Subsp. alnus Mill.

Rosaceae

R? 84-Potentilla erecta (L.) Raeusch.(=P.erectavar.maurorumMaire) 
RR 85- Prunus avium L. (=Cerasusavium (L.) Moench.)

RR 86- Pyrus communis Subsp. gharbiana (Trab.) Maire (=P. gharbiana Trab.).

Rununculaceae

R-E 87- Aquilegia vulgaris Subsp. cossoniana (maire et sennen) Dobignard (=A. nevadensis var litardieri Sennen. $=$ A. vulgaris subsp. viscosa sensu Maire. $=$ A. vulgaris var. cossoniana Maire et Sennen).

R 88- Delphinium staphisagria L.

RR 89- Helleborus foetidus L.

R 90-Ranunculus sceleratus L.

Scrophulariaceae

R? 91- Cymbalaria muralis subsp. muralis P.Gaertn.,B. Mey. \& Scherb.

P-E 92- Digitalis laciniata subsp. Riphaea (=D. abscura L. var riphaea Pau et font Quer)

C-V-E 93 - Digitalis mauretanica (Humbert et Maire ex Emb. Et Maire) Ivanina (=D. Purpurea var.Mauretanica Humbert \& Maire ex Emb\& Maire=Digitalis Purpurea var. mauretanica (Humbert\& Maire ex Emb\& Maire) Romo).

V 94- Scrophularia sambucifolia Subsp. mellifera (L'her. Ex aiton) Maire (=S. mellifera L'Her ex Aiton).

RR 95- Scrophularia sambucifolia subsp. Sambucifolia (=S. mellifera var .vidalii Pau= S. sambucifolia subvar. rubriflora Maire= S. sambucifolia subvar viridiflora ( Poir ) Maire= $S$.trisecta $\mathrm{Pau}=$ S.viridiflor $a$ Poir.)

\section{Solanaceae}

C $\quad 96$ - Atropa baetica Willk

RR 97-Atropa bella-donna L.

Sparganiaceae

RR 98- Sparganium erectum subsp. Erectum (= S. ramosum subsp. polyedrum Asch.et Graebn.)

\section{Tamaricaceae}

R? 99- Tamarix gallica L. (= T.brachystylis var.litteralis Pau et Font Quer $=T$. pulchellasennen)

Taxaceae

V 100- Taxus baccata L.

Urticaceae

RR 101- Urtica dioica L.

Violaceae

P 102- Viola arborescens L.

RR 103- Viola canina L.

RR-C 104- Viola munbyana var. rifana emb.et Maire (= V. munbyana var. rifana Emb. et Maire)

R 105- Viola reichenbachiana Jord ex Boreau (= V. sylvestris Lam.)

RR-C 106- Viola riviniana Rchb. (= V. sylvestris Lam.) 